\title{
Efficiency of ultrasonic treatment of polysaccharide from brown algae
}

\author{
Victoria E. Suprunchuk * (iD
}

North-Caucasus Federal University, 355017 Pushkin st., 1, Stavropol, Russia

* Corresponding author: vsuprunchuk@ncfu.ru

This short communication (letter) belongs to the MOSM2021 Special Issue.

(C) 2021, The Authors. This article is published in open access form under the terms and conditions of the Creative Commons Attribution (CC BY) license (http://creativecommons.org/licenses/by/4.0/).

\section{Abstract}

Ultrasonic exposure can be used for depolymerization of brown algae polysaccharides. However, its effectiveness depends on several factors, including cavitation activity in the treatment medium. Therefore, the purpose of the work was to determine the cavitation activity and the effectiveness of the ultrasonic exposure to fucoidan in order to optimize the processing processes of polysaccharide from brown algae. A change in cavitation activity was revealed depending on the composition of the processing environment, as well as on the intensity of ultrasonic exposure with a constant frequency of the ultrasonic wave. Similar dynamics of change of cavitation activity were established at the intensity of ultrasonic treatment of 100 and $133 \mathrm{~W} / \mathrm{cm}^{2}$ with amplification of electric signal at the increase of ultrasound intensity. The use of SDS in the processing medium led to an increase in cavitation activity to $14.9 \pm 0.47 \mathrm{mV}$. Treatment of the fucoidan solution for 40 minutes under various conditions allowed to obtain fractions with a change in the average hydrodynamic particle diameter from $113 \mathrm{~nm}\left(100 \mathrm{~W} / \mathrm{cm}^{2}\right)$ to $85 \mathrm{~nm}\left(200 \mathrm{~W} / \mathrm{cm}^{2}\right)$ and $124 \mathrm{~nm}$ (SDS).
\end{abstract}

\section{Keywords}

fucoidan

cavitation

nanoparticles

depolymerization

Received: 02.11.2021

Revised: 16.12.2021

Accepted: 20.12.2021

Available online: 23.12 .2021

\section{Introduction}

Fucoidans are of great interest among biopolymers of marine origin. Fucoidan is a branched sulfated heteropolysaccharide isolated from brown algae and some marine invertebrates [1]. Fucoidan has anticancer [2], antithrombic [3], anticoagulant [4], antioxidant [5], antiviral [6] activity and other pharmacologically important properties and its use is approved by the FDA. However, as a rule, this polysaccharide has a high molecular weight, which limits its industrial use. Therefore, we can make a conclusion that depolymerization of fucoidan is considered an urgent task. Ultrasonic treatment is often used in order to lower the molecular weight of polymers, in polysaccharides in particular $[7,8]$. This method is simple and environmentally friendly. Ultrasonic processing is based on the phenomenon of cavitation. When treated with ultrasonic, the cavities are formed in the environment - cavitation bubbles. As a result of the cavitation bubble collapse, a shock wave is created with the formation of an acoustic flow leading to the formation of turbulence due to the continuous formation and collapse of cavities in the system. In addition, shock waves, intense local heating (about $5000{ }^{\circ} \mathrm{C}$ ) and high pressure (about
$1000 \mathrm{~atm})$ are created [9]. As a result of the collapse of such bubbles, sufficient energy is released to break bonds in any polymeric materials [10].

However, the effectiveness of ultrasonic treatment depends on a number of factors, one of which is the cavitation activity in the treatment medium. Detection of cavitation activity will allow determining the optimal rate of destruction of the biopolymer. Therefore, the purpose of the work was to determine the cavitation activity and the effectiveness of ultrasonic treatment to fucoidan in order to optimize the processing of polysaccharide from brown algae. The measurement of cavitation intensity was based on recording acoustic noise as an electrical signal.

\section{Experimental}

For ultrasonic treatment, polysaccharide obtained from brown algae Fucus vesiculosus was used according to the procedure presented in [11] with some changes. Subsequent cleaning was carried out in accordance with the work of A.M. Urvantsev, I. Yu. Bakunin, N. Yu. Kim and others [12]. The resulting fucoidan was dispersed in deionized water at a concentration of $10 \mathrm{mg} / \mathrm{ml}$. 
The ultrasonic waves generated by means of the ultrasonic UIP10oohd processor with a power of $1 \mathrm{~kW}$ (Hielscher Ultrasonics GmbH, Germany) with a frequency of $20 \mathrm{kHz}$ induced through sonotrode. In the first case, the sonotrode was placed in an aqueous fucoidan solution and the ultrasonic intensity was varied to 100,133 , $200 \mathrm{~W} / \mathrm{cm}^{2}$. In the second case, the composition of the medium was varied by introducing auxiliary substances into an aqueous solution of fucoidan while maintaining the intensity of ultrasonic exposure. Surfactants (SDS, PEG-400) were used as excipients. Ultrasonic treatment of fucoidan was carried out for 40 minutes with constant cooling with the help of an ice bath with temperature control within the range of $45 \pm 5{ }^{\circ} \mathrm{C}$.

The size of the obtained particles was determined using Photocor Compact Z (Photocor LLC, Russia). A cavitometer was used in order to determine the intensity of cavitation. The principle of the cavitometer is based on the analysis of cavitation noise with its conversion to an electric signal. The hydrophone was placed in a treatment medium at a depth of $45 \pm 2 \mathrm{~mm}$ below the surface of the liquid and an electrical signal was recorded.

\section{Results and discussion}

Cavitation is the formation of bubbles experiencing local pressure fluctuations, the occurrence of which is possible under the influence of an ultrasonic wave [13]. Cavitation measurements were carried out in the work using a cavitometer, the action of which is based on processing the spectrum of cavitation noise received by a broadband hydrophone, followed by converting an acoustic signal into an electric one. The more intense the shock wave, the wider the spectrum of cavitation noise and the larger the electric signal. The measurement of cavitation activity in the form of an electrical signal were carried out during ultrasonic exposure of the fucoidan solution.
The work revealed that cavitation activity changes unevenly. This is because the volume fraction of the cavitation bubble plays an important role in cavitation. The large cavitation bubble in medium reduces acoustic transparency and can cause attenuation of the ultrasonic waves during their propagation [13]. However, when bubbles collapse, shock waves form, which can lead to an increase in acoustic emission.

Similar dynamics of change of cavitation activity were established at the intensity of ultrasonic exposure of 100 and $133 \mathrm{~W} / \mathrm{cm}^{2}$, as well as when used in the SDS processing medium. In addition, the amplification of the electric signal was revealed when the intensity of ultrasound increased. Therefore, in the first minute, the average value of this signal increased from $7.94 \pm 0.21 \mathrm{mV}$ (with an ultrasound intensity of $100 \mathrm{~W} / \mathrm{cm}^{2}$ ), $9.2 \pm 0.47 \mathrm{mV}$ (with an ultrasound intensity of $133 \mathrm{~W} / \mathrm{cm}^{2}$ ) to $10.4 \pm 1.35 \mathrm{mV}$ (with an ultrasound intensity of $200 \mathrm{~W} / \mathrm{cm}^{2}$ ). At higher ultrasound power, a cone-shaped bubble structure [14] is formed which can lead to the effect of screening and scattering of ultrasound. This leads to a change in the shape of the acoustic emission plot compared to the acoustic emission plots at lower values of sound wave intensity (Fig. 1).

Application in the treatment medium (SDS) showed an increase in cavitation activity up to $14.9 \pm 0.47 \mathrm{mV}$. The use of SAA PEG-400 also led to an increase in the electric signal relative to the medium without the use of SAA at the same ultrasonic wave intensity from 9.2 $\pm 0.47 \mathrm{mV}$ to $10.2 \pm 0.92 \mathrm{mV}$, but to a lesser extent than SDS. In general, during ultrasonic exposure, a decrease in cavitation is observed during the first $500 \mathrm{~ms}$.

When used in the SDS processing medium, there is a decrease in cavitation activity from $14.90 \pm 0.47 \mathrm{mV}$ to $11.00 \pm 0.21 \mathrm{mV}$ at the 30th minute of exposure (Fig. 2). It is known that SAA leads to a decrease in surface tension in liquids, as a result of which the number of collapse bubbles decreases and they accumulate [15].

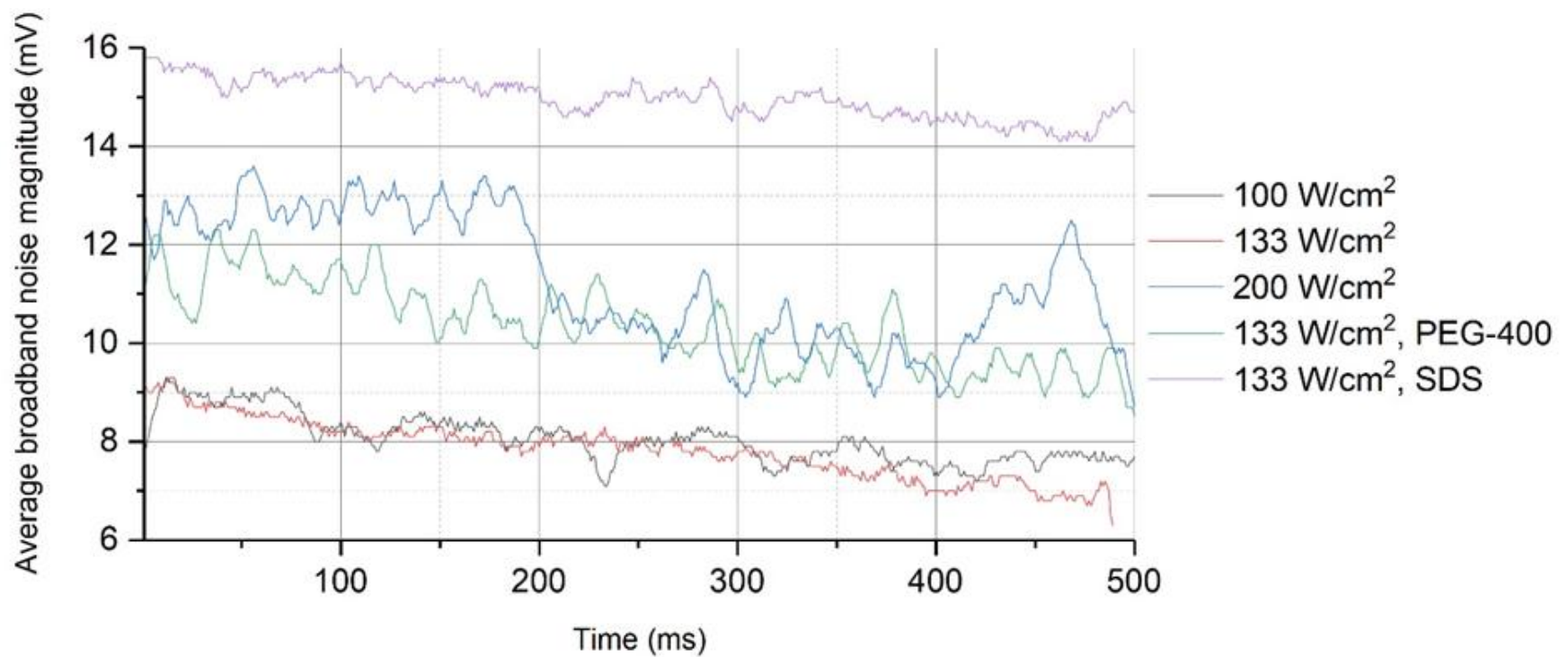

Fig. 1 Graph of the cavitation activity change at ultrasonic exposure during the first $500 \mathrm{~ms}$ 


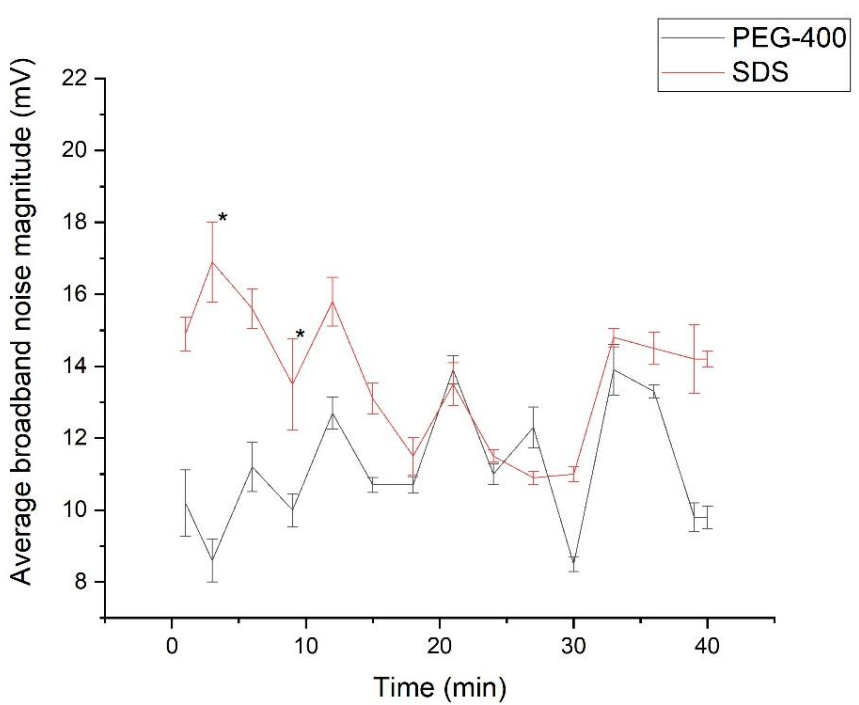

Fig. 2 Change in cavitation activity at ultrasonic exposure during 40 min when SAA is used (* - results of 5 measurements)

At the same time, the introduction of SAA prevents the Bjerknes force and leads to electrostatic repulsion of cavitation bubbles [16], thereby their fusion is prevented, growth slows down and the lifetime of the bubbles increases. The retention of bubbles, in turn, can block the transmission of sound through the liquid to the hydrophone [17], as a result of which the electrical signal is reduced. In general, there is a decrease in cavitation activity to $9.80 \pm 0.31 \mathrm{mV}$ when used in a processing medium PEG400 and to $14.20 \pm 0.22 \mathrm{mV}$ when using SDS.

SAAs have been used in the treatment environment under the assumption that their action caused forced conformational changes, which are formed during the movement of polymer chains. In turn, this makes it possible to adopt the unwound shape of the chain and increase its sensitivity to the shear force of the shock wave when the cavitation bubble collapses [18].

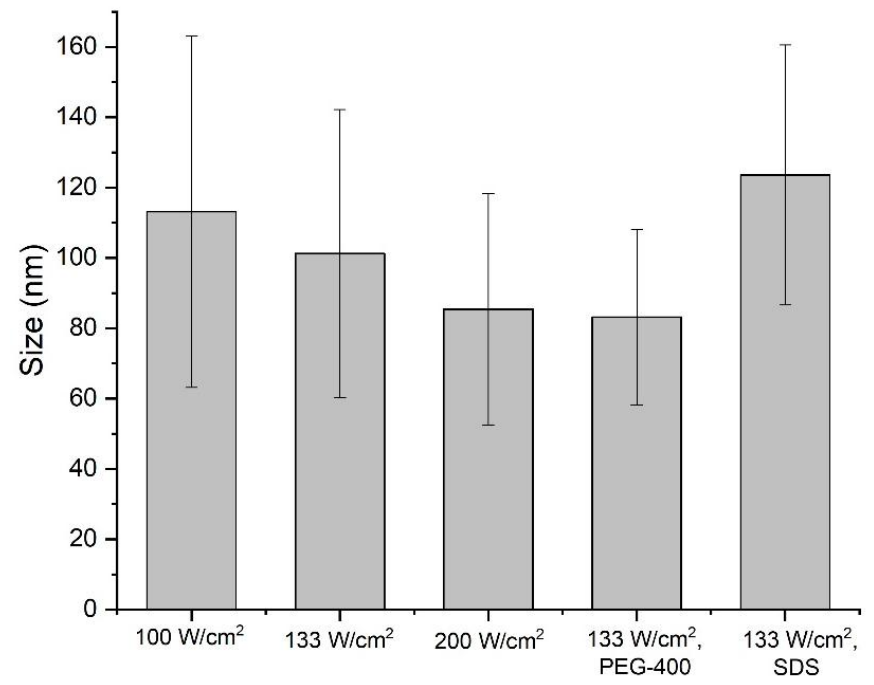

Fig. 3 Dependence of size of nanoscale fraction of fucoidan on parameters of ultrasound and composition of processing environment
According to Fig. 3, there is a decrease in the particle size of the polysaccharide of the nanoscale fraction of fucoidan with an increase in the intensity of ultrasonic exposure. The average hydrodynamic diameter of the obtained particles, as in the case of molecular weight, has a certain value for a given intensity. It is known that in branched polysaccharides of the form "tangle", chain break is more difficult than in linear "stick-shaped" macromolecules (for example, chitosan). This is due to the fact that linear conformation leads to the accumulation of "pulling forces" throughout the entire chain [19]. It is possible that this difficulty can be overcome by increasing the intensity of the ultrasonic wave or introducing PEG-40O, allowing to obtain fractions with an average particle size of $85 \pm 33$ and $83 \pm 25 \mathrm{~nm}$, respectively.

\section{Conclusions}

Cavitation activity in the treatment medium upon change of intensity of ultrasonic action and composition of the treatment medium was investigated. A direct proportional dependence of the ultrasound efficiency on the intensity of ultrasound wave was revealed. Similar dynamics of change of cavitation activity at intensity of ultrasonic action of 100 and $133 \mathrm{~W} / \mathrm{cm}^{2}$ with amplification of electric signal upon the increase of ultrasound intensity was established. In such a way, with an ultrasound intensity of $200 \mathrm{~W} / \mathrm{cm}^{2}$ the electric signal increased to $10.40 \pm 1.35 \mathrm{mV}$. The use of SDS in the processing medium led to an increase in cavitation activity to $14.90 \pm 0.47 \mathrm{mV}$. After 40 minutes of treatment of the fucoidan solution under various conditions, fractions with a change in average particle size from $113 \mathrm{~nm}\left(100 \mathrm{~W} / \mathrm{cm}^{2}\right)$ to $85 \mathrm{~nm}\left(200 \mathrm{~W} / \mathrm{cm}^{2}\right)$ and $124 \mathrm{~nm}$ (SDS) were obtained. Increasing the intensity of the ultrasonic wave or introducing PEG-400 allows obtaining fractions with an average particle size of $85 \pm 33$ and $83 \pm 25 \mathrm{~nm}$, respectively. Therefore, determination of ultrasonic impact efficiency will allow optimizing the technological process of fucoidan destructuring.

\section{Funding sources}

Scholarship of the President of the Russian Federation to young scientists and graduate students № SP-1758.2021.4 "Development of a nanobiocomposite tPA carrier for targeted high-performance thrombolytic therapy".

\section{References}

1. Wang Y, Xing M, Cao Q, Ji A, Liang H, Song S. Biological activities of fucoidan and the factors mediating its therapeutic effects : a review of recent studies. Mar Drugs. 2019;17:183. doi: $10.3390 / \mathrm{md} 17030183$

2. Jin JO, Chauhan PS, Arukha AP, Chavda V, Dubey A, Yadav D. The therapeutic potential of the anticancer activity of fucoidan: Current advances and hurdles. Mar Drugs. 2021;19:117. doi:10.3390/md19050265 
3. Cui K, Tai W, Shan X, Hao J, Li G, Yu G. Structural characterization and anti-thrombotic properties of fucoidan from Nemacystus decipiens. Int J Biol Macromol. 2018;120:1817-22. doi:10.1016/j.ijbiomac.2018.09.079

4. Colliec S, Fischer AM, Tapon-Bretaudiere J, Boisson C, Durand P, Jozefonvicz J. Anticoagulant properties of a fucoidan fraction. Thromb Res. 1991;64:143-54. doi:10.1016/0049-3848(91)90114-C

5. Wang S, Huang C, Chen C, Chang C, Huang C, Dong C, et al. Structure and biological activity analysis of fucoidan isolated from Sargassum siliquosum. ACS omega. 2020;5:32447-55. doi:10.1021/acsomega.0c04591

6. Wang W, Wu J, Zhang X, Hao C, Zhao X, Jiao G, et al. Inhibition of influenza A virus infection by fucoidan targeting viral neuraminidase and cellular EGFR pathway. Sci Rep. 2017;7:114. doi:10.1038/srep40760

7. Mason J, Cuthbert C, Brookfield A. Effect of ultrasound on the degradation of aqueous native dextran. Ultrason Sonochem. 1995;2:1-3. doi:10.1038/srep40760

8. Tiwari BK, Muthukumarappan K, Donnell CPO, Cullen PJ. Rheological properties of sonicated guar, xanthan and pectin dispersions. Int J Food Prop ISSN. 2010;13:223-33. doi: $10.1080 / 10942910802317610$

9. Suslik KS, Fang M., Hyeon T, Mdleleni M. Applications of sonochemestry to materials synthesis. Sonochemistry and Sonoluminescence. 1999;291-320.

10. Mason TJ, Newman AP, Phull S. Sonochemistry in water treatment. 2nd international conference on advances in water and effluent treatment. Professional Engineering Publishing. 1993. p. 243-50.

11. Zvyagintseva TN, Shevchenko NM, Popivnich IB, Isakov V V., Scobun AS, Sundukova EV, et al. A new procedure for the separation of water-soluble polysaccharides from brown seaweeds. Carbohydr Res. 1999;322:32-9. doi:10.1016/So0o8-6215(99)00206-2
12. Urvantseva AM, Bakunina IU, Kim NYU, Isakov VV, Glazunov $\mathrm{VP}, \mathrm{Zvy}$ agintseva TN. Isolation of purified fucoidan from a natural complex with polyphenols and its characteristics. Chem plant raw mater. 2004;15-24. Russian.

13. Frohly J, Labouret S, Bruneel C, Looten-Baquet I, Torguet R. Ultrasonic cavitation monitoring by acoustic noise power measurement. J Acoust Soc Am. 2000;108:2012-20. doi:10.1121/1.1312360

14. Moussatov A, Granger C, Dubus B. Cone-like bubble formation in ultrasonic cavitation field. Ultrason Sonochem. 2003;10:191-5. doi:10.1016/S1350-4177(02)o0152-9

15. Iwai Y, Li S. Cavitation erosion in waters having different surface tensions. Wear. 2003;254:1-9. doi:10.1016/So043-1648(02)00305-8

16. Wood RJ, Lee J, Wood RJ, Lee J, Bussemaker MJ. A parametric review of sonochemistry : Control and augmentation of sonochemical activity in aqueous solutions. Ultrason Sonochem. 2017;38:351-70. doi:10.1016/j.ultsonch.2017.03.030

17. Verhaagen B, Fernández Rivas D. Measuring cavitation and its cleaning effect. Ultrason Sonochem. 2016;29:619-28. doi:10.1016/j.ultsonch.2015.03.009

18. Caruso MM, Davis DA, Shen Q, Odom SA, Sottos NR, White SR, et al. Mechanically-induced chemical changes in polymeric material. Chem Rev. 2009;109:5755-98. doi:10.1021/cr9001353

19. Czechowska-Biskup R, Rokita B, Lotfy S, Ulanski P, Rosiak JM. Degradation of chitosan and starch by $360-\mathrm{kHz}$ ultrasound. Carbohydr Polym. 2005;60:175-84. doi:10.1016/j.carbpol.2004.12.001 